\title{
Electron microscope study of the advanced tendinopathy process of the long head of the biceps brachii tendon treated arthroscopically
}

\author{
J. Zabrzyński' ${ }^{1}$ M. Gagat² , Ł. Paczesny³ ${ }^{3}$ Ł. Łapaj4 ${ }^{4}$ D. Grzanka ${ }^{5,6}$ \\ ${ }^{1}$ Department of Orthopaedic Surgery, Multidisciplinary Hospital, Inowroclaw, Poland \\ ${ }^{2}$ Department of Histology and Embryology, University Hospital No. 1, Bydgoszcz, Poland \\ ${ }^{3}$ Department of Orthopaedic Surgery, Orvit Clinic, Torun, Poland \\ ${ }^{4}$ Department of General Orthopaedics, Musculoskeletal Oncology and Trauma Surgery, \\ Poznan University of Medical Sciences, Poznan, Poland \\ ${ }^{5}$ Department of Clinical Pathomorphology, University Hospital No. 1, Bydgoszcz, Poland \\ ${ }^{6}$ Department of Dermatology, Sexually Transmitted Infections and Immunodermatology, \\ Nicolaus Copernicus University, Bydgoszcz, Poland
}

[Received: 15 August 2017; Accepted: 19 October 2017]

Background: The ultrastructural alterations related to tendinopathy have not been well described. Most studies on this subject have been conducted many years ago and focused on material from the Achilles tendon. It was demonstrated that various comorbidities can affect ultrastructural alterations in the advanced tendinopathy; however, there is very little data on ultrastructural morphology in tendinopathies related to mechanical overload as in case of the long head of the biceps brachii tendon (LHBT). The aim was to determine intermediate ultrastructural alterations in middle to severe grade the LHBT tendinopathy and to establish if they are different than those reported in the literature for other anatomical locations.

Materials and methods: In this study we examined the ultrastructure of a series of the LHBT fragments arthroscopically removed due to tendinopathy and investigated the morphology of tenocytes and collagen fibres in cases of the LHBT tendinopathy.

Results: In pathological samples tenocytes were randomly scattered, their shape was round and the shape of nuclei was also disrupted. The presence of apoptotic-like features in tenocytes' nuclei was noted. All samples showed replacement of collagen fibrils by non-collagen extracellular matrix and diffuse collagen disorganisation.

Conclusions: It was demonstrated at ultrastructural level that the LHBT tendinopathy is not simply a wear and tear phenomenon, since chronic degeneration of the extracellular matrix and tenocytes were present, similarly as in tendinopathies, in other anatomical locations. (Folia Morphol 2018; 77, 2: 371-377)

Key words: biceps tendon, long head of the biceps brachii tendon (LHBT), tendinopathy, transmission electron microscopy (TEM), ultrastructure

Address for correspondence: J. Zabrzyński, MD, Multidisciplinary Hospital, Department of Orthopaedic Surgery, ul. Poznańska 97 , 88-100 Inowrocław, Poland, tel/fax: +48 5235454 78, e-mail: zabrzynski@gmail.com 


\section{INTRODUCTION}

Recent advances in understanding of tendons' mechano-biology and structure are approaching to the solution of mystery of the tendinopathy process $[1,3,17]$. Multiple studies demonstrated that the hierarchical structure of tendon is strictly associated with its function in human body and provides minimisation of the risk of failures and injuries. Macroscopically healthy tendons appear as a fibro-elastic, solid structures and are characterised by high mechanical strength [17]. The microscopic structure consists of extracellular matrix (ECM) and various types of cells, predominantly tenocytes which are responsible for synthesis of collagen molecules and support of the balance in the ECM. The ECM acts as scaffolding for cells, vessels, nerves and is responsible for the mechanical function of tendons; it consists predominantly of collagen bundles arranged in a parallel pattern [17]. Furthermore, serves as a network for transmission of information; integrins and cytoskeleton transduce mechanical stimuli created by loads and manipulate the tenocytes activity [17].

The basic structural unit of the tendon is the fibril, which is visible under the electron microscope (EM) [6]. Fibrils aggregate into fibres, characterised by unique curly architecture, known as "wavy configuration", seen in the light microscope (LM) studies [5]. Bundles of fibres, connected together, are forming larger structures, called fascicles. The number and diameter of fascicles depend on the type of muscle and loads it is subjected to, but it may also vary from tendon to tendon, even within the same tendon [5]. Superb biomechanical properties and strength of tendon tissue result from collagen fibrils density, length, orientation, inter-molecular cross-links and finally organisation of the ECM.

Tendinopathy is a chronic disorder of tendons, with multifactorial aetiology, common both in the athletic and non-athletic population. It is characterised by disruption of the hierarchical structure of the tissue and pathological alterations of cells, ECM, nerves, and vascular bed [18]. Degeneration of the tissue decreases the mechanical durability of tendons which may ultimately lead to ruptures. Currently magnetic resonance imaging (MRI) and ultrasound (US) studies allow for early identification of tendinopathy in many tendons, and minimally invasive techniques such as arthroscopy are used in some cases, such as tendinopathy of the long head of the biceps brachii tendon (LHBT) $[2,4]$. This pathology is a common source of pain in the anterior shoulder area, usually localised at the level of intertubercular groove of the humerus $[10,16]$. It is thought that up to $90 \%$ cases of the LHBT tendinopathy is accompanied by additional shoulder disorder, usually, rotator cuff tears (RCTs) $[4,16]$. It is therefore generally accepted that mechanical overload plays a key role in development of the LHBT tendinopathy, although the aetiology is not fully understood. This is in contrast to the Achilles tendon, where multiple factors lead to tendon pathology, and mechanical overload seems to be one of many contributors $[1,11]$.

The ultrastructural alterations related to tendinopathy have not been well described. Most studies on this subject have been conducted many years ago and focused on material from Achilles tendon either ruptured or with end-stage tendinopathy, which was related to limited imaging and treatment options available at that time. It was demonstrated that various comorbidities, such as diabetes can affect ultrastructural alterations in the advanced tendinopathy; however, there is very little data on ultrastructural morphology in tendinopathies related to mechanical overload as in case of the LHBT [8].

In this study we examined the ultrastructure of a series of the LHBT fragments arthroscopically removed due to tendinopathy and investigated the morphology of tenocytes and collagen fibres in cases of the LHBT tendinopathy. The aim was to determine intermediate ultrastructural alterations in middle to severe grade the LHBT tendinopathy and to establish if they are different than those reported in the literature for other anatomical locations, where mechanical overload is less important in development of disorders.

\section{MATERIALS AND METHODS}

This study included 4 consecutive patients treated arthroscopically, due to the LHBT tendinopathy. Subjects were recruited from the Department of Orthopaedic Surgery. The LHBT tendinopathy was diagnosed preoperatively and based on physical examination, non-contrast MRI scans and US. The mean age of the subjects was 54 years (49-62 years, standard deviation [SD] 14.3) and the cohort included 2 males and 2 females. Patients were nonathlete population, with no history of inflammatory or rheumatic diseases, suffering from painful disorders in the anterior area of the shoulder more than 1 year. All patients underwent a shoulder 
Table 1. Summary of arthroscopy, light microscope and transmission electron microscopy (TEM) investigation. Each patient had a concurrent pathology of the affected shoulder. Macroscopically each sample presented the same features of degenerative process. Light microscope study according to Bonar scale resulted in high score and confirmed advanced degeneration. Finally, the TEM investigation showed pathological fibrils morphology and arrangement with deeply deformed tenocytes

\begin{tabular}{lccccc}
\hline Patient no. & Age [years] & $\begin{array}{c}\text { Concomitant lesions } \\
\text { in the affected joint }\end{array}$ & $\begin{array}{c}\text { Macroscopic analysis } \\
\text { of tendon samples }\end{array}$ & $\begin{array}{c}\text { Bonar score } \\
\text { [points] }\end{array}$ & TEM alterations \\
\hline 1 & 62 & SST + SSC & Laceration & 10 & Disorganised architecture and abnormal \\
morphology of collagen fibrils
\end{tabular}

ACJ — acromio-clavicular joint; SI — subacromial impingement; SSC — subscapularis tendon tear; SST — supraspinatus tendon tear

arthroscopy-assisted biceps tenodesis, as previously described [2]. Portions of the intra-articular part of the LHBT, which are remnants after arthroscopic treatment, were harvested from each of patients who underwent a procedure. The intra-articular part of the tendon was removed by arthroscopic scissors about $1 \mathrm{~cm}$ from the origin point - at the supraglenoid tubercle - to the bicipital groove area. The residual part at the supraglenoid tubercle was removed by vaporisation technique. The resected fragments were sent to the Department of Histology and Embryology Nicolaus Copernicus University, fixed in sterile 10\% buffered formalin, in a transport container. Samples were dehydrated in increasing concentrations of ethanol, cleared in xylene, embedded in paraffin, cut into sections of $4 \mu \mathrm{m}$ thickness by microtome, floated onto glass slides. We used haematoxylin and eosin staining for routine histopathological examination using Eclipse E800 light microscope (Nikon, Tokyo, Japan); samples were scored using Bonar system, where 0 is given for normal tendons, while 12 corresponds to maximal damage $[13,15]$.

Before the intra-articular part of the LHBT was embedded in formalin, small-size sample (maximum $4 \mathrm{~mm}$ in diameter), was biopsied from the central part of the intra-articular fragment and fixed with $2.5 \%$ buffer glutaraldehyde. After washing with $0.1 \mathrm{M}$ sodium cacodylate buffer, the samples were postfixed in $2 \%$ osmium tetroxide in $0.1 \mathrm{M}$ cacodylate buffer for $1 \mathrm{~h}$ room temperature and then passed through a graded series of ethanol and acetone solutions. After infiltration with resin, the samples were embedded in Epon 812 (Sigma-Aldrich, St. Louis, MO, USA). Ultra-thin sections were cut by ultramicrotome and collected on copper grids (Sigma-Aldrich, St.
Louis, MO, USA), double-stained with uranyl acetate and lead citrate. The samples were examined using a JEM 100CX (JEOL, Tokyo, Japan) transmission electron microscopy (TEM) operating at $60 \mathrm{kV}$.

Since obtaining samples of healthy biceps tendon for use as controls is very difficult due to bioethical concerns, we decided to obtain samples of healthy tendon tissue from subjects who undergo anterior cruciate ligament $(A C L)$ reconstruction using thigh tendons and did not manifest pathologies of semitendinosus or gracillis tendons (STG). Control samples obtained from 4 patients undergoing arthroscopic reconstruction of the $A C L$ with mean age of 37 years (4 males, age $24-49$ years, SD 10.2). We used fragments obtained away from the enthesis to assure that only tendinous tissue was harvested; preparation of the STG samples we performed exactly in the same way as it was in the LHBTs.

The study was approved by local bioethics committee (approval no. KB 598/2016), all patients were volunteers and gave informed consent. The study has been carried out in accordance with The Code of Ethics of the World Medical Association (Declaration of Helsinki) for experiments involving humans.

\section{RESULTS}

Preoperative physical examination revealed pain in the anterior shoulder area in all subjects with the duration of symptoms 1-5 years. Arthroscopic evaluation showed additional pathologies, such as RCTs in 2 patients, subacromial impingement (SI) in 2 patients, acromio-clavicular joint (ACJ) degeneration in 2 patients (Table 1 ). The macroscopic evaluation of the harvested LHBT portions revealed heterogeneous morphology, with features of laceration, fraying, widening and flattening of the resected fragments of 


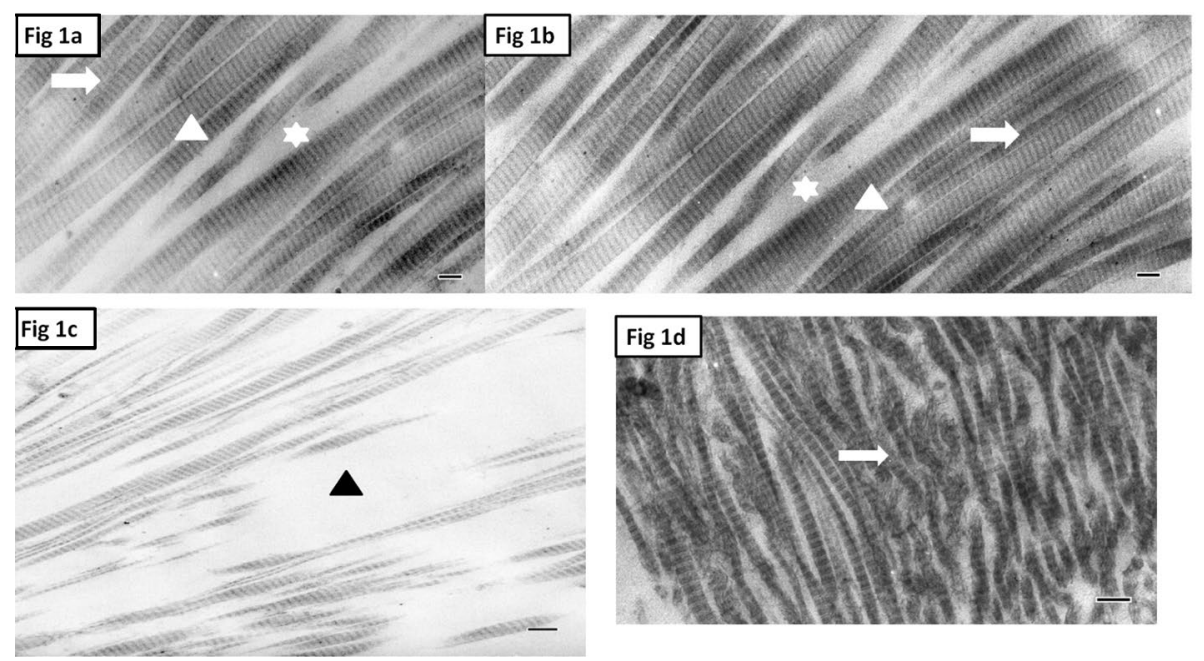

Figure 1. A, B. Transmission electron microscopy (TEM) of normal, control tendons, demonstrate collagen fibrils, with axial and parallel orientation (arrow), with organised architecture and characteristic banding pattern (arrowhead). Non-collagen extracellular matrix (ECM) is reduced to minimum (asterisk). Original magnification panel $\mathbf{A}$ and $\mathbf{B} \times 8300$; bar $=200 \mathrm{~nm}$. C, D. TEM of pathological tendons samples, perform a longitudinal sections of pathological long head of the biceps brachii tendon with disrupted architecture of collagen fibrils, loss of axial and parallel arrangement (arrow), when compared to panel $\mathbf{A}$ and $\mathbf{B}$. Panel $\mathbf{D}$ presents predominant loss of collagen fibrils with abundant development of the ECM (arrowhead). Original magnification panel $C \times 8300$; panel $D \times 5000$, bar $=200 \mathrm{~nm}$.

tendon. Samples obtained from STG, control group, showed homogenous morphology and appeared as a solid, white-brilliant structure with no sign of tear or fraying.

Light microscope studies demonstrated a variety of pathological, structural changes among the LHBT samples. In the examined cohort mean Bonar score was 8.75 (range 8-10; SD 0.95) which indicates intermediate to advanced tendinopathy in these cases. In all samples the morphology of tenocytes was altered, with an increased number of magnified, rounded cells, with enlarged nuclei and a highly reduced amount of cytoplasm. Loss of characteristic organisation scheme was also present in all cases; the longitudinal alignment of collagen fibres was distorted and the characteristic "crimping pattern" was not present. Moreover, the separation of collagen bundles by abundant ground substance was present, with accumulated ground substance between them. Finally, the ingrowth of new capillary vessels forming clusters was noted. In the control samples of the STG a low number of fusiform and spindle-like tenocytes were found. The characteristic "wavy configuration" of collagen fibres was preserved with their axial orientation. Only a few capillary vessels were found and there was no vascular expansion. The Bonar score for all of these samples was 0 .

The TEM investigation was focused on arrangement of fibres in several planes, as described in the

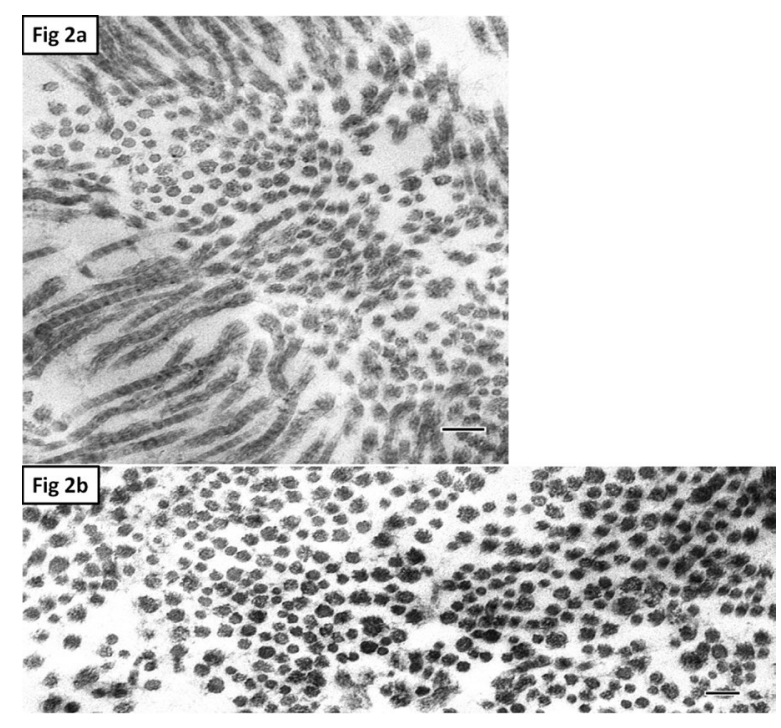

Figure 2. A, B. Transmission electron microscopy of pathological tendons samples, demonstrate transverse and oblique sections of the harvested long head of the biceps brachii tendon with cylindrical shape fibrils, not differentiated into large and small diameter fibrils. Original magnification panel $A \times 8300$, panel $B \times 7000$, bar $=200 \mathrm{~nm}$.

literature $[5,9,14]$. In case of the LHBT samples an abnormal morphology of collagen fibrils was revealed in all cases (Fig. 1C, D). Transverse and oblique sections showed a distinct decrease of their diameter (up to $150 \mathrm{~nm}$ ) and reduction of their profile dimensions (Fig. 2). These changes were seen in most fibrils, although occasionally large-diameter fibrils 


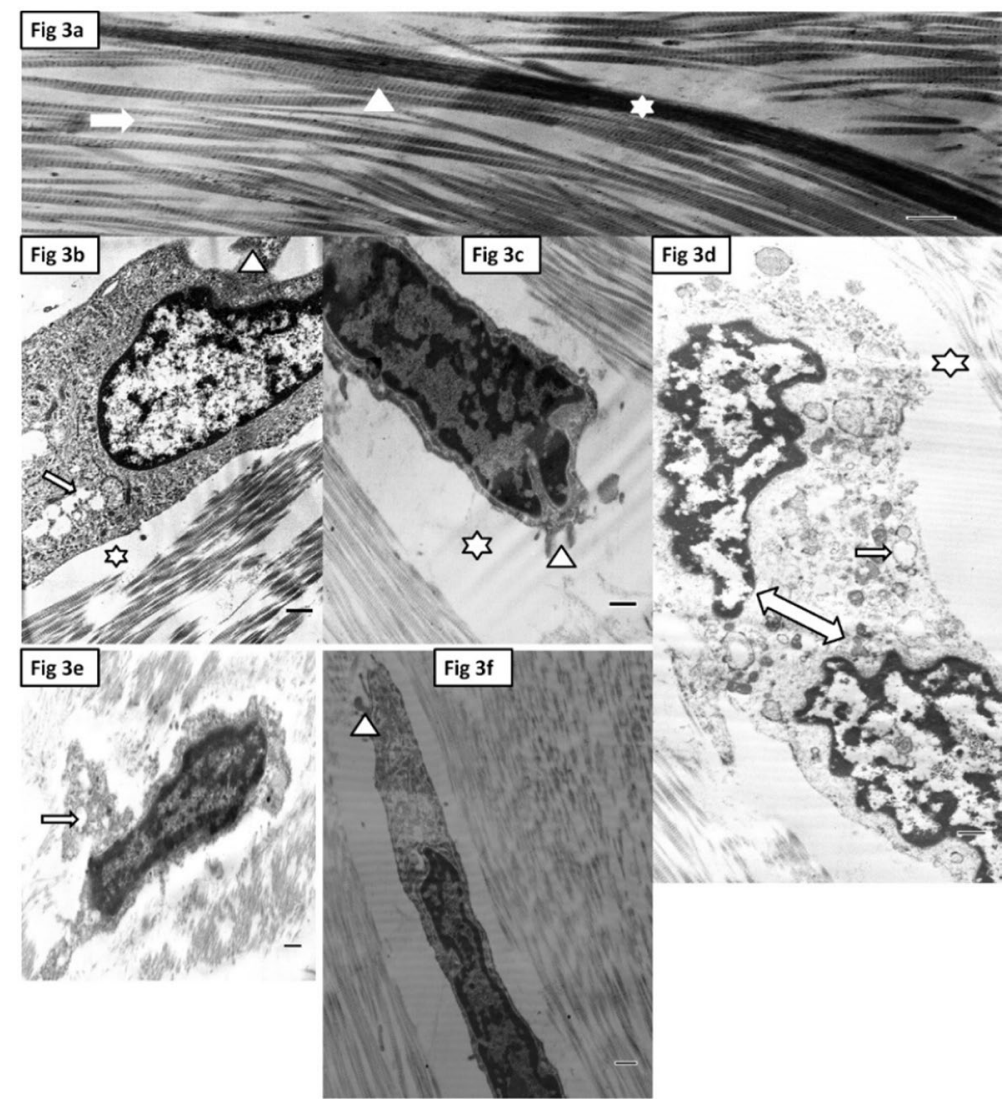

Figure 3. A. Transmission electron microscopy (TEM) of normal, control tendon showing tenocyte with characteristic elongated, regular, fusiform shape. Tenocyte is arranged parallel to collagen net. The cytoplasm is reduced to minimum and the cell is dominated by the nucleus (asterisk); collagen fibrils are arranged parallel (arrow). There is no characteristic of pathological tendons pericellular non-collagen space or surface between cells and collagen fibrils. Tenocyte adheres close to collagen fibrils (arrowhead). Original magnification panel $A \times 4000$, bar $=500 \mathrm{~nm}$. C, D, E, F, G. TEM of pathological tendons samples showing malformed tenocytes of the long head of the biceps brachii tendon samples. The shape of tenocytes is rounded (B, C, D), more irregular than in healthy tendon, with prominent cytoplasm and well-developed pericellular matrix. There is increased pericellular space of non-collagen extracellular matrix. Asterisk indicates thickened pericellular matrix. Interior of some cells is rich in numerous vacuoles, vesicles, and mitochondria (arrows) (B, D). These features confirm intensive metabolic processes of the cells. Cytoplasmic processes of tenocytes are deeply disturbed when compared with tenocytes from literature data (arrowheads) (B, C, F). In few tenocytes we found features of apoptosis process. Nuclear chromatin becomes dense and abundant (E). Panel $\mathbf{D}$ presents fragmentation of the nucleus and chromatin (double-headed arrow). Original magnification panels $\mathrm{C}-\mathrm{F} \times 5000$. were found, randomly scattered within the tissue. These samples were characterised by disorganised architecture; fibrils did appear as a homogenous mass, instead they were scattered randomly without the characteristic parallel, axial pattern; large amounts of non-collagen ECM were visible in all cases (Fig. 1C, D). In contrast, control samples during TEM investigation were characterised by parallel arrangement of fibrils and showed a diversified range of fibrils' cross-links with frequent presence of larger diameter fibrils and compact arrangement of fibres on transverse crosssections (Fig. 1A, B).

In the LHBT samples, tenocytes, embedded in a network of collagen fibrils, were enlarged as compared to control tendons. The shape of cells was deformed; their characteristic elongation seen in control samples was lost (Fig. 3B-F). Moreover, there was noticed an accumulation of vacuoles inside tenocytes' cytoplasm and vacuoles of waste products near the cellular lamina (Fig. 3B, D). Characteristic cytoplasmic processes of tenocytes, which are responsible for penetration of the ECM, were deeply deformed when compared with tenocytes from the control group (Fig. 3C, F). Instead, in most tenocytes from the LHBT samples there was a pericellular halo formed by noncollagen ECM, separating them from the collagen fibrils (Fig. 3B-F). In a few tenocytes of one subject the features of apoptosis were found: the marginalisation of chromatin and nuclear fragmentation, moreover, apoptotic blebs were seen (Fig. 3D, E).

\section{DISCUSSION}

The LHBT has a complex biomechanics and is involved in forearm supination, elbow flexion, humeral head depression and is a weak abductor of the shoulder $[2,12]$. In recent years, numerous investigators have noticed the correlation between the LHBT disorders and RCT tears, superior labral tear from anterior to posterior, SI and ACJ degeneration [2, 4]. Therefore it is generally believed that the LHBT pathology is typically a secondary process, related to others shoulder lesions. This is in contrast to Achilles tendon, where the development of tendinopathy is multifactorial and mechanical overload is only one of contributors [1]. Since most studies regarding ultrastructural changes in tendinopathy focused on Achilles tendon, we decided to investigate if they are also seen in tendinopathy related to mechanical overload, as in the case of LHBT. 
For this reason we studied the intra-articular part of the LHBT which is especially prone to friction and compressing forces leading to microtrauma.

Similarly as previous reports regarding tendinopathy in humans, our study is limited in several ways. The cohort was small and not homogenous, similarly as in other ultrastructural studies, still it must be underlined, that findings of this study may not apply to all cases of LHBT tendinopathy [14]. Due to ethical reasons we were unable to obtain samples of LHBT from healthy live donors; that is why the hamstring tendons were included from healthy individuals as controls. These tendons have a relatively large enthesis at pes anserinus, that is why we obtained only fragments away from it in order to preserve normal tendon morphology. Our LM assessment was based on semiquantitative scoring system which can be affected inter- and intra-observer differences. To minimise this all samples were examined by 3 authors, similarly as in other studies.

Transmission electron microscopy studies of healthy tendons demonstrated hierarchical structure of the ECM and a small number of tenocytes equipped with long, slender cytoplasmic processes dedicated for ECM infiltration [5]. Their 3-dimensional organisation is characterised by the even arrangement of cells located parallel to the long axis of collagen bundles. A distinct feature of tendons is the characteristic "crimping pattern" of collagen fibres. Crimps serve as a shock absorbers and thus temporary deformation allows for dissipation of the energy [6]. Franchi et al. [6] have investigated crimps morphology in relaxed and stretched Achilles tendon. Examination of the ultrastructure of collagen fibrils using the TEM showed that each of them contained numerous knots which can be considered as the "fibril crimps". These knots are a locations where fibrils are bending or twisting and changing their direction. During stretching, fibre crimps undergo straightening under the LM, but fibrillar crimps under TEM are intact. These fibrillar crimps are an actual aspect of shock absorbers. However, some differences between various anatomical location exist, especially with regard to the diameter of fibrils; it was found that increased diameter of fibrils provides increased strength of tissue [5]. Hill et al. [9] analysed the ultrastructure of labrum and anchor of the LHBT using TEM and noticed heterogeneous composition of their structures. They noted the distinct mechanical function of these structures due to various fibril diameter.
Most TEM studies regarding tendinopathy focused on the Achilles tendon and included samples from ruptured tendons and very advanced tendinopathy cases. One of most characteristic findings of these studies was a decrease in fibril diameter. In one study a reduction of diameter from $241 \mathrm{~nm}$ to $246 \mathrm{~nm}$ in healthy regions to $150-154 \mathrm{~nm}$ in tendinopathic regions was demonstrated [14]. Grant et al. [8] demonstrated accumulation of small-diameter fibrils (range 35-160 nm) in Achilles tendons of patients with diabetes mellitus. This was similar to our study, where small fibrils with a diameter of less than $150 \mathrm{~nm}$ were commonly seen in LHBT samples, while large fibrils were seen in controls (up to $250 \mathrm{~nm}$ diameter). The increased ratio of small diameter to large diameter fibril in pathological portions may have an influence on their structure, susceptibility to further damage and progress of the tendinopathy process [14].

The abnormal aggregate of collagen fibril or alterations in the diameter and cross-sectional profile are common features of pathological tissue. This phenomenon is also common in tumours, but masses of disorganised collagen were found in a study of human tendon samples $[10,16]$. Collagen disorganisation leads to tissue heterogeneity and loss of architecture. Galliani et al. [7] in their study of 11 patients who suffered from lateral epicondylitis found areas of highly disorganised ECM, where fibrils were fragmented into short segments. This data was consistent with our LM and TEM results. In the pathological tendon tissue, there is a loss of crimps and either loss of mechanical buffer. Our LHBT samples showed replacement of collagen fibrils (in LM and TEM), by non-collagen ECM and subsequently decrease in a number of crimps.

Our observations of the ultrastructure of tenocytes revealed a few characteristic alterations. Similarly as in other studies tenocytes were randomly scattered, their shape was round, localisation and the shape of nuclei was also disrupted [14]. The transformation of the shape of tenocytes, into more oval shape, could be an evidence of cartilage metaplasia and adaptation; the intra-articular part of the LHBT is exposed more to friction and compression forces than traction. It is thought that cytoplasm and cellular shape variations reflect the gradual chondroid transformation [13]. The metaplasia of fibroblasts into chondrocytes is characteristic for advanced tendinopathy [13].

Some authors noted the presence of apoptoticlike features appearance in tenocytes' nuclei, charac- 
terised by chromatin alterations and fragmentation $[7,17]$. Increased numbers of apoptotic cells were observed in pathologies of the supraspinatus, patellar, and tibialis anterior tendons. Tenocytes of our one subject tendon had features of apoptosis; however, based on data from this and other study we cannot determine if it is caused by tendinopathy, related to ageing or related to other factors.

\section{CONCLUSIONS}

Since over $90 \%$ of the LHBT tendinopathy cases are associated with other shoulder lesions, it is believed that mechanical overload seems to be the main pathology in this condition. However, in this study we demonstrated at ultrastructural level that the LHBT tendinopathy is not simply a wear and tear phenomenon, since chronic degeneration of the ECM and tenocytes were present, similarly as in tendinopathies, in other anatomical locations. A surprising finding of this study is presence of apoptotic alterations in tenocytes. Since we are unable to explain their cause, we believe that this aspect of tendinopathy should be explored in future studies.

\section{Acknowledgements}

Authors are grateful to the staff of the Department of Histology and Embryology for their technical assistance.

\section{REFERENCES}

1. Abate M, Silbernagel KG, Siljeholm C, et al. Pathogenesis of tendinopathies: inflammation or degeneration? Arthritis Res Ther. 2009; 11(3): 235, doi: 10.1186/ar2723, indexed in Pubmed: 19591655.

2. Ahrens PM, Boileau P. The long head of biceps and associated tendinopathy. J Bone Joint Surg Br. 2007; 89(8): 1001-1009, doi: 10.1302/0301-620X.89B8.19278, indexed in Pubmed: 17785735.

3. Andarawis-Puri N, Flatow EL, Soslowsky $\sqcup$. Tendon basic science: Development, repair, regeneration, and healing. J Orthop Res. 2015; 33(6): 780-784, doi: 10.1002/ jor.22869, indexed in Pubmed: 25764524.

4. Ditsios K, Agathangelidis F, Boutsiadis A, et al. Long head of the biceps pathology combined with rotator cuff tears. Adv Orthop. 2012; 2012: 405472, doi: 10.1155/2012/405472, indexed in Pubmed: 23209915.
5. Franchi M, Fini M, Quaranta M, et al. Crimp morphology in relaxed and stretched rat Achilles tendon. J Anat. 2007; 210(1): 1-7, doi: 10.1111/j.1469-7580.2006.00666.x, indexed in Pubmed: 17229278.

6. Franchi M, Trirè A, Quaranta M, et al. Collagen structure of tendon relates to function. ScientificWorldJournal. 2007; 7: 404-420, doi: 10.1100/tsw.2007.92, indexed in Pubmed: 17450305.

7. Galliani I, Burattini S, Mariani AR, et al. Morpho-functional changes in human tendon tissue. Eur J Histochem. 2002; 46(1): 3-12, indexed in Pubmed: 12044045.

8. Grant WP, Sullivan R, Sonenshine DE, et al. Electron microscopic investigation of the effects of diabetes mellitus on the Achilles tendon. J Foot Ankle Surg. 1997; 36(4): 272-278; discussion 330, indexed in Pubmed: 9298442.

9. Hill AM, Hoerning EJ, Brook $\mathrm{K}$, et al. Collagenous microstructure of the glenoid labrum and biceps anchor. J Anat. 2008; 212(6): 853-862, doi: 10.1111/j.14697580.2008.00904.x, indexed in Pubmed: 18429974.

10. Joseph M, Maresh CM, McCarthy MB, et al. Histological and molecular analysis of the biceps tendon long head post-tenotomy. J Orthop Res. 2009; 27(10): 1379-1385, doi: 10.1002/jor.20868, indexed in Pubmed: 19340876.

11. Kaux JF, Forthomme B, Goff CLe, et al. Current opinions on tendinopathy. J Sports Sci Med. 2011; 10(2): 238-253, indexed in Pubmed: 24149868.

12. Longo UG, Loppini M, Marineo G, et al. Tendinopathy of the tendon of the long head of the biceps. Sports Med Arthrosc Rev. 2011; 19(4): 321-332, doi: 10.1097/ JSA.0b013e3182393e23, indexed in Pubmed: 22089281.

13. Maffulli N, Longo UG, Franceschi F, et al. Movin and Bonar scores assess the same characteristics of tendon histology. Clin Orthop Relat Res. 2008; 466(7): 1605-1611, doi: 10.1007/ s11999-008-0261-0, indexed in Pubmed: 18437501.

14. Pingel J, Lu Y, Starborg T, et al. 3-D ultrastructure and collagen composition of healthy and overloaded human tendon: evidence of tenocyte and matrix buckling. J Anat. 2014; 224(5): 548-555, doi: 10.1111/joa.12164, indexed in Pubmed: 24571576.

15. Singaraju VM, Kang RW, Yanke AB, et al. Biceps tendinitis in chronic rotator cuff tears: a histologic perspective. J Shoulder Elbow Surg. 2008; 17(6): 898-904, doi: 10.1016/j. jse.2008.05.044, indexed in Pubmed: 18786837.

16. Streit JJ, Shishani Y, Rodgers M, et al. Tendinopathy of the long head of the biceps tendon: histopathologic analysis of the extra-articular biceps tendon and tenosynovium. Open Access J Sports Med. 2015; 6: 63-70, doi: 10.2147/ OAJSM.S76325, indexed in Pubmed: 25792859.

17. Xu Y, Murrell GAC. The basic science of tendinopathy. Clin Orthop Relat Res. 2008; 466(7): 1528-1538, doi: 10.1007/ s11999-008-0286-4, indexed in Pubmed: 18478310.

18. Zabrzyński J, Zabrzyńska A, Grzanka D. Tendinopathy a disease of tendons. J Orthop Trauma Surg Related Res. 2016; 3(40): 24-30. 